\title{
Gemeinschaft der Schöpfung auf dem Weg in die Zukunft Gottes
}

\section{Jürgen Moltmanns Denken angesichts der neuen Enzyklika "Laudato si “ von Papst Franziskus}

\author{
Sr. Monika Amlinger OSB
}

\section{Zusammenfassung}

Es werden Grundgedanken des reformierten Theologen Jürgen Moltmann zur Theologie der Schöpfung untersucht. Es handelt sich einerseits um den Gedanken der Gemeinschaft aller Geschöpfe, nicht nur der Menschen, andererseits um den Blick auf die eschatologische Zukunft der ganzen Schöpfung. Es wird dargestellt, welches neue Naturverständnis Moltmann entwickelt und wie er die Macht des Menschen über die Natur deutet. Er beschreitet neue theologische Wege, indem er alle Geschöpfe in den Blick nimmt. Es zeigt sich aber, dass er die Gemeinschaft der Geschöpfe zu wenig differenziert darlegen kann, was u. a. mit seiner einseitigen Konzentration auf den Heiligen Geist in der Schöpfung zusammenhängt. Es zeigen sich inhaltlich Parallelen zu Gedanken von Papst Franziskus in der Enzyklika „Laudato si'“, wobei dieser mehr den göttlichen Vater in den Mittelpunkt stellt, vor dem alle Geschöpfe Söhne und Töchter bzw. Geschwister seien. Auch der Papst scheint die Vollendung aller Geschöpfe zu lehren.

\section{$1 \quad$ Gemeinschaft in Gott und die Ausrichtung auf die verheißene Zukunft der Schöpfung}

Am 24. Mai 2015 veröffentlichte Papst Franziskus die erste Umweltenzyklika ${ }^{1}$ der römisch-katholischen Kirche mit dem Titel „Laudato si ““, die der Sorge um nachhaltige Entwicklung auf dem Planeten Erde gewidmet ist. Dort schreibt er:

1 In Wahrheit ist die neue Enzyklika „Laudato si “ (= LS) nicht eine reine Umweltenzyklika, sondern ein Schreiben, das die Interdependenzen des Umgangs mit der nichtmensch-

(C) Der/die Autor(en) 2017

K.-D. Altmeppen et al. (Hrsg.), Nachhaltigkeit in Umwelt,

Wirtschaft und Gesellschaft, DOI 10.1007/978-3-658-14439-5_7 
Die Geschöpfe dieser Welt können nicht als ein herrenloses Gut betrachtet werden: Alles ist dein Eigentum, Herr, du Freund des Lebens (Weish 11,26). Das gibt Anlass zu der Überzeugung, dass sämtliche Geschöpfe des Universums, da sie von ein und demselben Vater erschaffen wurden, durch unsichtbare Bande verbunden sind und wir alle miteinander eine Art universaler Familie bilden, eine sublime Gemeinschaft, die uns zu einem heiligen, liebevollen und demütigen Respekt bewegt. (LS 89)

Er spricht hier - in Anklang an den Sonnengesang des Heiligen Franziskus, der die anderen Geschöpfe als „Brüder“ und „Schwestern“ bezeichnet (LS 1f.87) - von einer großen „Familie“ und „Gemeinschaft“, die zwischen allen Lebewesen und Dingen in unserer bekannten Welt bestehe. Diese Familie gründet für ihn in Gott, dem „Vater“. Von diesem Vater schreibt er an späterer Stelle, er sei „der letzte Ursprung von allem, der liebevolle und verbindende Grund von allem, was existiert“ (LS 238). Die Verbindung zwischen allem, was ist, besteht für ihn also in ihrem gemeinsamen Urgrund. Des Weiteren führt er aus, die Welt sei „nach göttlichem Bild erschaffen“, als „Gewebe von Beziehungen“, da auch Gott in Beziehungen (von Vater, Sohn und Geist), als „Communio“, lebe. ${ }^{2}$ Für Papst Franziskus hat diese Erkenntnis praktische Auswirkung: sie bewegt uns zu Respekt gegenüber allem, was lebt und existiert. Nichts ist für ihn daher gleichgültig, ein „herrenloses Gut“, alles hat eine Bedeutung vor Gott, dem Vater, ist sogar sein „Eigentum“ und sollte entsprechend auch wahrgenommen und behandelt werden.

Die Gedanken des Papstes in der Enzyklika weisen deutliche Gemeinsamkeiten, aber auch einige charakteristische Unterschiede zum Werk des weltbekannten reformierten Theologen Jürgen Moltmann auf. Moltmann ist einer derjenigen Theologen, die sich innerhalb der Schöpfungstheologie zum ersten Mal auch mit ökologischen Fragen - ausgehend von der sog. „ökologischen Krise“ - beschäftigten. Im Jahr 1985 veröffentlichte er sein Hauptwerk zur Schöpfungstheologie „Gott in der Schöpfung. Ökologische Schöpfungslehre“, in dem er eine christliche Theologie der Schöpfung vorstellte. ${ }^{3}$ In diesem Aufsatz sollen Grundgedanken Moltmanns

lichen Schöpfung („Umwelt“) und des Umgangs mit (besonders) den benachteiligten Menschen (den Armen) in den Blick nimmt. Allerdings sieht Papst Franziskus die anderen Geschöpfe nicht nur als auf den Menschen hingeordnet, sondern in ihrem „Eigenwert“ vor Gott und den Menschen (LS 69), der in der Liebe Gottes zu jedem Geschöpf gründe (LS 57.246). Zur Enzyklika: Manzke, 2015 und Marx, 2015.

2 LS 239; 240. „Für die Christen führt der Glaube an den einen Gott, der trinitarische Communio ist, zu dem Glauben, dass die gesamte Wirklichkeit in ihrem Innern eine eigentlich trinitarische Prägung besitzt“ (LS 239).

3 Durch die sehr dialogische Ausrichtung seines Denkens und die Einbeziehung von Einsichten besonders auch aus der orthodoxen Tradition (z. B. der Gedanke der pericho- 
vorgestellt und diskutiert werden ${ }^{4}$, die die aktuelle Betrachtung der Thesen von Papst Franziskus vertiefen und ergänzen können. ${ }^{5}$

Es sind vor allem zwei Grundthemen Moltmanns, die hier betrachtet werden sollen und die eine Parallele zum Denken des Papstes darstellen: zum einen der Gedanke der Gemeinschaft in Gott selbst und in der Schöpfung (bzw. zwischen beiden), zum anderen die Ausrichtung auf die verheißene Zukunft der Schöpfung (Neuschöpfung), ausgehend von der Auferstehung Jesu von den Toten. ${ }^{6} \mathrm{Im} \mathrm{Zu}-$ sammenhang dieser Thesen entwickelt Moltmann ein neues Naturverständnis und eine ungewohnte Sicht der Stellung des Menschen in der Natur, aber auch eine Analyse der gegenwärtigen „ökologischen Krise“ bzw. „Katastrophe“ und damit des Leidens der Schöpfung. ${ }^{7}$

retischen Gemeinschaft Gottes) wirkte Moltmann prägend bzw. herausfordernd nicht nur auf die evangelische Theologie, sondern auf Theologen nahezu aller Konfessionen.

4 Die hier vorgestellten Gedanken Moltmanns stammen vor allem aus seinem Hauptwerk zur Schöpfungstheologie „Gott in der Schöpfung“, aber auch aus der Trinitätstheologie („Trinität und Reich Gottes“) und Eschatologie („Das Kommen Gottes“) (Moltmann, 1980; 1985; 1995).

5 Es kann in diesem Aufsatz kein detaillierter Vergleich zwischen den Gedanken des Papstes und der Schöpfungstheologie Moltmanns geleistet werden. Der Schwerpunkt liegt auf der Darstellung der Einsichten bzw. Thesen Moltmanns. Zum Ganzen der Schöpfungstheologie Moltmanns, besonders auch in ihrer trinitarischen (gemeinschaftlichen) und eschatologischen Ausrichtung: Pehar, 2006.

6 Auch in der Beurteilung der Ursachen und (v. a. sozialen) Zusammenhänge der gegenwärtigen ökologischen Krise zeigen sich deutliche Ähnlichkeiten, die aber hier höchstens, wenn überhaupt, in Fußnoten angedeutet werden.

7 Die Theologie Moltmanns ist primär pastoral und existentiell ausgerichtet, indem er von der Theodizeefrage und vom Gedanken des Mit-Leidens Gottes mit seiner Schöpfung ausgeht. Die Aufgabe der Theologie besteht für ihn darin, das „Überleben mit [der] offenen Wunde“ zu ermöglichen (Moltmann, 1980: 65). Moltmann, geb. am 8. April 1926, schreibt über die biographischen Hintergründe seiner Theologie:

Ich fragte nach existenztragendem Wissen und verlor das Interesse an naturerkennendem und naturbeherrschendem Wissen. Ich brauchte ,Trost im Leben und Sterben', wie der Heidelberger Katechismus sagt, und fand ihn durch das zufällige Lesen der Bibel und die unverdiente Freundlichkeit schottischer und englischer Christen in dem Christus, der in seiner Passion mein Bruder wurde und durch seine Auferstehung von den Toten auch mich zu einer lebendigen Hoffnung erweckte. Meine Todeserfahrungen am Ende des Krieges, meine Depressionen über die Schuld meines Volkes und die inneren Gefahren der völligen Resignation hinter Stacheldraht waren der erste locus theologicus für mich und sind es im tiefsten Grunde meiner Seele auch geblieben. (Moltmann, 1999: 20) 


\section{2 "Gemeinschaftliches" Denken}

\subsection{Gemeinschaft in Gott - gemeinschaftliche Schöpfung}

Für Moltmann zeigt sich die Relevanz des Schöpfungsglaubens „in Vorstellung und Wegen aus der ökologischen Krise der Gegenwart“ (Moltmann, 1985: 36). Diese Wege hängen bei ihm wesentlich mit dem Gedanken der Gemeinschaft zusammen. ${ }^{8}$ Ausdrücklich schließt er seine Schöpfungslehre an seine Trinitätslehre an, in welcher er Gott als Gemeinschaft vorstellt. ${ }^{9}$ Gott ist für ihn Gemeinschaft von Vater, Sohn und Geist, die als göttliche Personen in perichoretischer Verbundenheit, d.h. in vollständiger gegenseitiger Durchdringung und dem wechselseitigen Austausch ihres Lebens, existieren (Moltmann, 1980: 191f.). ${ }^{10}$ Diese perichoretische Gemeinschaft, in der Vater, Sohn und Geist in einem Lebensaustausch stehen (Moltmann, 1985: 23ff. $)^{11}$, sei für die ganze Schöpfung prägend. ${ }^{12}$

8 Christian Link spricht in Bezug auf die Schöpfungstheologie Moltmanns - gründend in dessen Trinitätslehre - von einer „hermeneutischen Entscheidung“, die er als „Schlüssel“ für das Verständnis dieser Theologie bezeichnet: „An die Stelle des absoluten (,monotheistischen') und souveränen Gottes, der das Verhältnis zur Welt als ein ,einseitiges Herrschaftsverhältnis' etabliert hat, tritt der trinitarische Gott der vollkommenen Gemeinschaft von Vater, Sohn und Geist, der stattdessen ein ,mehrstelliges Gemeinschaftsverhältnis` begründet.“ (Link, 1987: 83). Für Link ist die zweite „Fundamentalentscheidung"Moltmanns, die sich aus der oben formulierten ergibt, diejenige für die Weltimmanenz Gottes statt seiner Welttranszendenz. (Link, 1987: 84)

9 „Wurde dort eine soziale Trinitätslehre entwickelt, so geht es hier um die entsprechende ökologische Schöpfungslehre.“ (Moltmann, 1985: 16). Das Wort „ökologisch“verbindet Moltmann primär mit dem griechischen Ursinn als „Lehre vom Haus“. Damit meint er ,in einem tieferen Sinn“ die wechselseitige Einwohnung (Perichorese) von Gott und Schöpfung im Heiligen Geist (Moltmann, 1985: 12).

10 Dabei versteht er Personen und Relationen als komplementär, als sich wechselseitig ermöglichend (Moltmann, 1980: 189): so ist es kein Tritheismus, den er vertritt - wo die Personen unabhängig voneinander bestehen würden -, sondern eine Einheit der göttlichen Personen als „Einigkeit“ (Moltmann, 1980: 191ff.). Greshake (2001: 171) sieht die „Gefahr eines gewissen Tritheismus“ bei Moltmann.

11 Die Analogie zwischen Gott und Schöpfung ist bei Moltmann aber immer vermittelt durch die Eschatologie (s. u. Kap. 3)! Moltmann schreibt z. B. (1985: 246, Hervorheb. i. O.) zur menschlichen Gottebenbildlichkeit: „Die soziale Analogie betrifft hier die göttliche Gemeinschaft, die durch das gegenseitige Einwohnen des Vaters im Sohn und des Sohnes im Vater durch den Geist gebildet wird. Sie meint hier nicht die Vaterschaft oder die Sohnschaft, sondern die innertrinitarische Gemeinschaft. Die Relationsebene der Trinität wird in der imago Trinitatis irdisch dargestellt, nicht die trinitarische Konstitutionsebene."

12 Wie später in diesem Aufsatz deutlich wird, versteht Moltmann diese Prägung nicht im Sinne eine Analogie (z. B. analogia entis) in der creatio originalis, sondern als ein 
Moltmann legt in seiner Trinitätslehre dar, in Bezug auf die Schöpfung gehe ,alle Aktivität" vom Vater aus. Die Schöpfung gehe aus der Liebe des Vaters zum Sohn hervor, die über diese gleichsam hinausreiche und nach einer freien geschöpflichen Erwiderung (in Entsprechung zum Sohn) suche. Der Sohn ist für Moltmann auch die „prägende Ursache“ der Schöpfung.

In seiner Schöpfungslehre, die auf die Trinitätslehre folgt, ist es dann aber primär der Geist Gottes, aus dem die Schöpfung hervorgeht und der sie auf ihre Zukunft hin entwirft. Im göttlichen Geist wird für Moltmann die Nähe bzw. Immanenz Gottes in der Schöpfung erfahrbar und verständlich, die herauszustellen ihm ein Grundanliegen ist. ${ }^{13}$ Durch die starke Betonung der Immanenz Gottes im Unterschied zu seiner Transzendenz (die Moltmann eher mit Vater und Sohn in Verbindung bringt) konzentriert sich Moltmann auf den Heiligen Geist, aus dessen Energien für ihn die Gemeinschaft der Schöpfung hervorgeht. Im Heiligen Geist entsteht für Moltmann die Gemeinschaft der Schöpfung.

Ist der Heilige Geist auf die ganze Schöpfung ,ausgegossen', dann schafft er die Gemeinschaft aller Geschöpfe mit Gott und untereinander zu jener Schöpfungsgemeinschaft,

Wirken des göttlichen Geistes gleichsam aus der Zukunft der Schöpfung heraus, auf ihre Vollendung hin. Obwohl Moltmann auch von der „Konstitution“ der Dreieinigkeit Gottes spricht, dem Hervorgang des Sohnes und des Geistes aus dem Vater, von dem sie ihr göttliches Wesen empfangen, blendet er in seinen Gedanken zur Einheit Gottes und auch zur Theologie der Schöpfung diesen Aspekt nahezu vollständig aus. Grund dafür ist sehr wahrscheinlich seine Ablehnung einseitiger hierarchischer Beziehungen, welche möglicherweise aus den (einseitigen) innergöttlichen Hervorgängen abgeleitet bzw. mit diesen in Verbindung gebracht werden könnten. Deshalb - so wird später dargestellt - kann Moltmann die „Herrschaft“ des Menschen über die anderen Geschöpfe entsprechend auch nicht als eine wirkliche Überordnung, sondern lediglich als Dienstfunktion verstehen.

13 Ausdrücklich legt Moltmann dar, er wolle eine „pneumatologische Schöpfunglehre“ entfalten (Moltmann, 1985: 12). „Alles, was ist, existiert und lebt vom andauernden Zufluss der Energien und Möglichkeiten des kosmischen Geistes. ... Durch die Energien und Möglichkeiten des Geistes ist der Schöpfer selbst in seiner Schöpfung präsent.“ (Moltmann, 1985: 23). Salai Hla Aung (1998: 175) zieht die Konsequenz, die Schöpfung müsse nach Moltmann nicht um ihrer selbst willen, sondern als Zeichen des Respektes Gott gegenüber (als des Immanenten) geachtet werden. Er kann nicht nachweisen, dass Moltmann wirklich so denkt, und so ist seine Schlussfolgerung als übertrieben anzusehen. Die beiden Aspekte müssen sich ja nicht ausschließen. Die Theologie des Heiligen Geistes bei Moltmann ist sehr oft kritisiert worden, vor allem was die Fundamentalunterscheidung von Gott und Welt angeht, was teilweise schlicht an terminologischen Unklarheiten oder Ungenauigkeiten Moltmanns liegt. Darauf weist z. B. auch Pehar (2006: 259f.) hin, die Moltmann in ihrer Dissertation über seine Schöpfungstheologie ansonsten kaum kritisiert. Dem kann hier nicht weiter nachgegangen werden. 
in der alle Geschöpfe auf je eigene Weise miteinander und mit Gott kommunizieren. ... Alles ist, lebt und webt in anderen, ineinander, miteinander, füreinander in den kosmischen Zusammenhängen des göttlichen Geistes. (Moltmann, 1985: 25)

Die "Ausgießung“ des Geistes, von der Moltmann hier spricht, ist ein eschatologisches Motiv, welches er aber für die creatio originalis zu verwenden scheint. ${ }^{14}$ Der Geist bringt die Schöpfung in Hinordnung auf ihre Vollendung hervor; er wirkt so gleichsam aus der Zukunft der Schöpfung heraus, wie im Folgenden noch deutlicher wird. Wie Gott für Moltmann wesentlich wechselseitiger Lebensaustausch unter den göttlichen „Personen“ ist, so ist auch die Welt bzw. Schöpfung für ihn als „Schöpfungsgemeinschaft" auf wechselseitigen Austausch des Lebens und nicht auf einseitige Machtausübung angelegt. ${ }^{15}$ In der von Gott verheißenen Zukunft, in der die Schöpfung ganz in diesem wohnen wird, wird sich nach Moltmann die Entsprechung zwischen Schöpfer und Schöpfung vollenden.

\subsection{Gemeinschaftliche Erkenntnis der Schöpfung}

Moltmann sucht nach einem originär theologischen Erkennen der Natur, einer zu entwickelnden „ökologischen Theologie der Natur“ (Moltmann, 1985: 47). Nur eine Erkenntnis, die selbst nicht bloß objektivierend, sondern welche "teilnehmend“ sei, führt dabei für ihn zu einer vertieften Schöpfungsgemeinschaft, zu welcher er mit seiner Schöpfungslehre beitragen will. ${ }^{16}$

„Eine Schöpfungslehre in ökologischer Hinsicht muss darum bemüht sein, das analytische Denken mit seinen Subjekt-Objekt-Distinktionen zu verlassen und ein neues, kommunikatives und integrierendes Denken zu lernen. Sie wird dabei auf den

14 Vielleicht ist es besser, bei Moltmann gar nicht von einer creatio originalis zu sprechen, sondern von verschiedenen "Stufen“ einer creatio nova (wobei dann das nova seinen eigentlichen Sinn verliert), auch wenn er selbst an einzelnen Stellen beide Ausdrücke verwendet und unterscheidet.

15 Auch im obigen Zitat von Papst Franziskus (LS 89) klingt dieses Thema der Herrschaft an, wenn er schreibt, die Geschöpfe seien kein „herrenloses Gut“ (das sich die Menschen als Herren aneignen könnten, wie sie dies faktisch tun), sondern sie seien „Eigentum“ Gottes - des eigentlichen Herren.

16 Zu diesem Ziel Moltmanns siehe Moltmann, 1985: 18. Zur gemeinschaftlichen Erkenntnis liest man auch: „Will man also das Wirkliche als das Wirkliche und das Lebendige als das Lebendige verstehen, dann muss man es in seiner ursprünglichen und eigenen Gemeinschaft, in seinen Beziehungen, Verhältnissen und Umgebungen erkennen.“ (Moltmann, 1985: 17). 
vormodernen Begriff der Vernunft als vernehmendes und anteilnehmendes Organ (methexis) zurückkommen müssen. (Moltmann, 1985: 17, 18)

Es geht Moltmann also darum, sich in der Erkenntnis wirklich betreffen zu lassen vom jeweils anderen und sich so mit den Dingen bzw. Lebewesen selbst zu verbinden. Moltmann hebt hervor, dass für ihn Schöpfungstheologie nicht - wie bisher üblich - nach der Erkenntnis Gottes aus der Schöpfung fragt, sondern nach der Erkenntnis der Schöpfung von Gott her. ${ }^{17}$ Im Licht des Glaubens erscheint ihm die Schöpfung als Schöpfungsgemeinschaft, die in wechselseitigem Austausch lebt. Naturgemäß treten demgegenüber die anderen Seiten der Schöpfung - zumindest im Werk über die Schöpfungstheologie - deutlich in den Hintergrund, wie Leid, Gewalt und Sünde. Leider gelingt es Moltmann auch nicht, gut zwischen verschiedenen Arten von Gemeinschaftlichkeit in der Schöpfung zu unterscheiden, vor allem zwischen der von Menschen möglichen und der zwischen Menschen und der übrigen Schöpfung.

\section{$3 \quad$ Die Zukunft Gottes}

\subsection{Gott ist im Kommen}

Moltmann versteht den dreieinigen Gott weniger als Ur-Sprung - und damit die Schöpfung als creatio originalis - denn als Zukunft, als den Zu-Kommenden der Schöpfung. Da Moltmann, wie bereits gesehen, den göttlichen Geist primär in eschatologischer Perspektive versteht ${ }^{18}$ und die Schöpfung wiederum primär vom Geist her deutet, ist Gott für ihn wesentlich der Zu-Kommende der Schöpfung, der in ihr immer mehr Wohnung nehmen will und in der Vollendung endgültig in ihr wohnt. ${ }^{19}$ Der „innere Grund“ des Bestehens der Schöpfung sei das „Reich

17 „Nicht was Natur zur Gotteserkenntnis beiträgt, sondern was der Gottesbegriff zur Naturerkenntnis beiträgt, soll untersucht werden." (Moltmann, 1985: 66). Das Genus der natürlichen Theologie sei Israel fremd und stamme aus der griechischen Philosophie, fügt er hinzu.

18 Im Geist wird die Schöpfung in die Verherrlichung des Vaters durch den Sohn hineingenommen und darin verklärt. Moltmann spricht auch von der Verherrlichung des Vaters und des Sohnes durch den Geist. (Moltmann, 1980: 140ff.).

19 Moltmann (1995: 22) legt dar, er wolle den „Advent“ als eschatologische Kategorie vertreten. Gott sei nicht der ewig Gegenwärtige, sondern der Kommende (vgl. auch den Titel des Werkes „Das Kommen Gottes“). Die Zukunft habe gegenüber Vergangenheit 
der Herrlichkeit“ (Moltmann, 1985: 68); sie sei von Anfang an auf ihre eigene Vollendung bezogen.

Diese Gedanken werden auch im Zeitverständnis Moltmanns deutlich:

Das ,Eschaton“ ist weder das Futur der Zeit noch die zeitlose Ewigkeit, sondern die Zukunft und Ankunft Gottes. Wir verwenden dafür einen adventlichen Begriff der Zukunft. ... Wir entfalten ihn philosophisch in einem Zeitverständnis, das die Zukunft als Ursprung und Quelle von Zeit überhaupt auffasst. (Moltmann, 1995: 39) ${ }^{20}$

Das Kommen Gottes zur Welt habe für diesen selbst eine große Bedeutung: er suche Ruhe in seinem endgültigen Wohnen in der Schöpfung (Moltmann, 1995: 13. 293). ${ }^{21}$ Denn mit seinem Schöpfungsentschluss habe Gott sich selbst an das Schicksal der Schöpfung gebunden und leide ihre Leiden mit, so dass die Vollendung der Schöpfung für ihn selbst eine Erlösung bedeute (Moltmann, 1980: 36ff.; 1985: 92ff.; 1995: 350ff.).

In der Vollendung der Schöpfung, die mit der Kategorie des „Novum“ zu erfassen ist, würden alle Geschöpfe eingesammelt; nichts gehe verloren. Moltmann vertritt eine Allversöhnungslehre, die er durch die Treue Gottes zu seinem Schöpfungswerk

und Gegenwart Priorität (Moltmann, 1995: 35). Sicouli (2006: 209f., Hervorheb. i. O.) stellt heraus, dass „,Neuschöpfung' als eine bzw. die hermeneutische Schlüsselkategorie im Werk Jürgen Moltmanns“ zu bewerten sei. Er spricht auch von einem „quasi postulatorische[n] ,Prinzip Neuschöpfung““ bei Moltmann. Haudel (2006: 288) merkt zu dieser eschatologischen Orientierung Moltmanns kritisch an: „So besteht die Gefahr, den metaphysischen Erweis Gottes aus der Welt lediglich durch ein eschatologisch-futurisches Verifikationsschema zu ersetzen, das den Gottesbegriff der eschatologischen Erfüllung der Heilsgeschichte unterwirft.“ Der Ausdruck „futurisch“ ist zwar verständlich, aber im Kontext des Moltmannschen Denkens nicht glücklich, da Moltmann unter dem „Futur“ im Unterschied zum „Advent“ bzw. zur (dt.) „Zukunft“ die Zukunft innerhalb der uns bekannten Schöpfung versteht und nicht die Neuschöpfung. „Zukunft als Futur verstanden ist das, was aus Vergangenheit und Gegenwart wird. Sie ist eine Form im Werdeprozess der physis. ... Gottes Sein ist im Kommen, nicht im Werden." (Moltmann, 1985: 143f., Hervorheb. i. O.). Den von Moltmann gesetzten einseitigen Akzent auf das eschatologische Verständnis der Schöpfung kann man mit seiner Ausblendung bzw. Vernachlässigung der innertrinitarischen Konstitution in Verbindung bringen.

20 Moltmann (1995: 43) bezeichnet die Zukunft (Gottes) auch als „Bedingung der Möglichkeit von Zeit“ und „Quelle der Zeit“. Baukham (2001: 157) stellt heraus, dass Moltmann die eschatologische Zukunft als gegenüber jeder Geschichte transzendent versteh. „On the necessity to break this continuity between history and eschatology ... Moltmann is in full agreement with Barth and Bultmann .... The eschatological future is related to history as future, but transcends all history.“

21 Dabei denkt Moltmann (1985: 279ff.) stark vom jüdischen Verständnis des Sabbat her, der eine reale Vorwegnahme der Ruhe Gottes in der Vollendung der Schöpfung sei. 
und die Kreuzestheologie begründet (Moltmann, 1995: 264ff.). ${ }^{22}$ Alle Geschöpfe stünden dann mit Gott und untereinander in inniger perichoretischer Gemeinschaft. Die Geschöpfe fänden einerseits ihre vollkommene Ruhe, andererseits ihre „ewige Lebendigkeit" (Moltmann, 1985: 220). Moltmann (1985: 20. 220) spricht auch von der „Sympathie aller Dinge“. ${ }^{23}$

\section{2 „Messianische“ Erkenntnis im Licht der Zukunft}

Für Moltmann wird die Wirklichkeit erst durch die heilsgeschichtliche Offenbarung als Schöpfung erkennbar. Das spezifisch christliche Erkennen der Schöpfung ist für Moltmann „messianisches“ Erkennen, also Erkennen im Licht des Messias Jesus Christus. Im Blick auf Jesus Christus werde einerseits das Leid der menschlichen und außermenschlichen Schöpfung sichtbar, andererseits weise der Glaube an die Auferstehung Jesu von den Toten ${ }^{24}$ und seine Herrlichkeit beim Vater auf eine Hoffnung für alle Geschöpfe. Für Moltmann (1995: 285ff.) sind alle Geschöpfe letztlich nicht der Vergänglichkeit ausgeliefert, sondern zu einer endgültigen neuen Existenz bei Gott - sogar in Gott - bestimmt.

Moltmann (1985: 70) scheint die Möglichkeit einer natürlichen Theologie - unabhängig von der geschichtlichen Offenbarung - einerseits zu leugnen, andererseits spricht er aber doch von ihr als einer „Erinnerung an ursprüngliche Gotteserkenntnis“. ${ }^{25}$ Die „natürliche Theologie“, welche Moltmann für möglich und sinnvoll

22 „... die einzig realistische Konsequenz aus der Kreuzestheologie ist die Wiederbringung aller Dinge“ (Moltmann, 1995: 278). Zur Erläuterung schreibt er (1995: 278): „Nicht der optimistische Traum einer geläuterten Menschheit, sondern die Höllenfahrt Christi [damit meint Moltmann die Gottverlassenheit Jesu, des Sohnes Gottes, am Kreuz, Anm. d. Verf.] begründet die Zuversicht, dass nichts verloren geht, sondern alles wiedergebracht und ins ewige Reich Gottes versammelt wird."

23 Es beginne die „ewige Zeit“, in der es keine Vergänglichkeit und keinen Tod mehr gebe (Moltmann, 1985: 220; 1995: 40.325).

24 Vgl. z. B. Moltmann (1985: 69): „Unter der Voraussetzung des Glaubens an Jesus Christus wird die Welt im messianischen Licht als geknechtete und zukunftsoffene Schöpfung offenbar."

25 Sie sei zugleich eine Vorwegnahme der Erkenntnis Gottes in der Herrlichkeit und habe somit eine „eschatologische Funktion“ (Moltmann, 1985: 71). Die „Spuren“ der anfänglichen Schöpfung, die sich finden ließen, seien zugleich „Abglanz“ der Herrlichkeit, welche die Christen erwarteten (Moltmann, 1985: 72). Auf den letzten Gedanken legt er ein deutliches Gewicht. 
hält, meint nicht ein Leuchten der Schöpfung aus sich heraus, in dem sie auf ihren Schöpfer als Ursprung hinweist, sondern ist primär ein Vorschein aus der Zukunft! ${ }^{26}$

„Dieser Vorschein hat den Charakter des messianischen Lichtes, das die gegenwärtige Welt in ihrer Bedürftigkeit offenbar und in ihrer Sehnsucht nach Freiheit lebendig macht und sie als reales Gleichnis und Verheißung des Reiches erkennen lässt.“ (Moltmann, 1985: 72).

Moltmann bindet also jede Erkenntnis der Welt als Schöpfung - in seiner evangelischen Prägung - an die geschichtliche Offenbarung Gottes, sowohl alt- wie auch, besonders, neutestamentlich.

Der Geist erfüllt für ihn alle Geschöpfe und den Kosmos als Ganzen mit „Selbsttranszendenz", die sich auf die Transzendenz Gottes hin ausrichtet und aus dieser heraus erst besteht (Moltmann, 1985: 112ff., 211ff.; 2010: 136).

Die Schöpfungserkenntnis von Jesus Christus her beinhaltet für Moltmann (1985: 73): „Im messianischen Licht werden alle irdischen Dinge und Lebewesen in ihrem Verfall an die Vergänglichkeit und in ihrer Hoffnung auf Befreiung zur Ewigkeit erkennbar." Er verweist hierbei auf Röm 8,19ff., wo Paulus diesen Zusammenhang formuliert. Die ethische Relevanz des christlichen Schöpfungsglaubens ist also, dass in seinem Licht einerseits das Leid der Geschöpfe, ihre „Knechtschaft“, andererseits - und vor allem - ihre Ausrichtung auf Vollendung hin erkennbar werden (Moltmann, 1985: 20, Hervorheb. i. O.). ${ }^{27}$ Die Gemeinschaft der Schöpfung ist so eine Gemeinschaft des Leidens, aber auch des Hoffens auf und der Sehnsucht nach auf gemeinsamer Vollendung. ${ }^{28}$

26 Moltmann (1985: 76) bezeichnet die Geschöpfe sogar als „Realschiffren“bzw. „Realverheißungen" ihrer eigenen Vollendung. Hier ergeben sich jedoch Fragen: einerseits legt er dar, die Schöpfung sei „gleichnisfähig und gleichnisbedürftig für ihre eigene Zukunft, das Reich Gottes, nicht aber für Gott selbst“. Er fährt fort, erst in der Vollendung werde sie „zu seinem Bild und Gleichnis“ (Moltmann, 1985: 75). Man kann nun fragen: wenn die Schöpfung jetzt schon ihre eigene Zukunft spiegelt, real antizipiert, ist sie dann nicht in diesem Sinne auch jetzt schon „Bild und Gleichnis“ Gottes?!

27 Dann stellt sich endlich auch die wahre Gemeinschaft der Geschöpfe untereinander ein: eine Gemeinschaft, die von den messianischen Traditionen des Judentums und des Christentums als die ,Sympathie aller Dinge bezeichnet wurde: Das Band der Liebe, der Teilnahme, der Mitteilung und der vielfältigen Wechselbeziehungen bestimmen das Leben der einen, im kosmischen Geist vereinigten Schöpfung. Es entsteht eine vielseitige Schöpfungsgemeinschaft.

28 Weiterhin ist Moltmann (1985: 83f.) - das kann hier nicht weiter entfaltet werden - der Gedanke wichtig, dass die Geschöpfe gemeinsam im Lobpreis Gottes stehen. Dies ist auch ihre eschatologische Berufung. Moltmann spricht hier von der „eucharistischen Gemeinschaft der Schöpfung“. Der Mensch ist für ihn zwar in besonderer Weise eucha- 


\section{$4 \quad$ Ein neues Naturverständnis - eine neue Sicht auf den Menschen}

\subsection{Die Einheit von Natur und Geschichte}

Aus dem gemeinschaftlichen Denken, das auf Zukunft, auf das Kommen Gottes hin orientiert ist, folgt bei Moltmann eine Neubestimmung des Verhältnisses von Natur und Geschichte. Sein Anliegen ist, sie in ihrer Einheit zu betrachten. Im weitesten Sinne versteht Moltmann dann unter der "Natur“ die ganze aktuell erfahrbare Schöpfung. ${ }^{29}$ Wie bereits gesehen, ist der „innere Grund“ der Schöpfung für Moltmann nicht der „Bund“ - so bei Karl Barth -, sondern das eschatologische

ristisches, zur (stellvertretenden) Danksagung berufenes und befähigtes Wesen, aber auch alle anderen Geschöpfe preisen Gott auf ihre Weise, sogar auch stellvertretend für den Menschen.

29 Moltmann folgt in Bezug auf sein Denken über das Verhältnis von Geschichte und Natur den Reflexionen Georg Pichts (Sicouli, 2006: 192f.).

,Natur' wird nun als Geschichte, Mensch und alle Geschöpfe umfassender Rahmen begriffen und ,Neuschöpfung' als universales Bild des Eschatologie. ... . Steht in Hinblick auf diese Vermittlung [von Geschichte und Natur] das frühere ... Werk [Moltmanns] im Zeichen der Hervorhebung der Geschichte unter Ausblendung der Schöpfung als Kosmos und Natur, so steht die spätere Reflexion im Zeichen der Aufwertung der Letzteren bis hin zu einer Subsummierung von Mensch und Geschichte in der Natur - und entsprechend des Reiches Gottes in der ,Neuschöpfung' als Symbol der kosmischen Eschatologie (Sicouli, 2006: 193).

Sicouli (2006: 192) führt aus, es lasse sich bei beiden Autoren „eine ähnliche Verschiebung“ des Denkens bzgl. der Verhältnisbestimmung von Natur und Geschichte feststellen. Moltmann (1985: 206) verweist in diesem Zusammenhang auch ausdrücklich auf Picht. Sicouli legt dar, Picht erweitere den Begriff der Natur auf die Bedeutung: ,alles, was in der Zeit ist“ (Sicouli, 2006: 193; FN 41; Picht, 1989). Nach Steven Bouma-Prediger zielt Moltmann nicht auf eine Neudefinition von Natur und Geschichte, sondern auf eine neue Bestimmung ihres Verhältnisses.

Thus it is unclear, dispite his contentions to the contrary whether he actually intends to rethink the very relata of the relationship itself. For example, he often uses the terms in traditional ways that suggest precisely the kind of dualism which he wishes to move beyond. In short, while Moltmann wishes to supercede the traditional paradigm of history, it is not clear that he goes as ,deep' as he thinks he does or as deep as he must. (Bouma-Prediger, 1995: 233)

Dazu ist - mit Sicouli - zu sagen, dass durch die Moltmannsche Ausdehnung des Naturbegriffes auf die ganze (aktuell erfahrbare) Schöpfung (so auch Bouma-Prediger, 1995: 233) keine Dualität entsteht, sondern eher eine Inklusion der Geschichte in die Natur. Ob Moltmann dieses Konzept immer begrifflich durchhalten kann, sei hier dahingestellt. 
Reich Gottes (Moltmann, 1985: 68). ${ }^{30}$ Insofern ergibt sich eine Einheit von Natur und Geschichte in ihrer Ausrichtung auf die gemeinsame Vollendung. ${ }^{31}$

Wir nehmen wahr, dass die Schöpfung auf Geschichte ausgerichtet ist, aber ihren letzten Sinn doch noch nicht darin findet, nur ein Schauplatz für die Geschichte Gottes mit den Menschen zu sein. Denn der letzte Sinn dieser Geschichte liegt seinerseits in der neuen, vollendeten Schöpfung. (Moltmann, 1985: 69)

Bereits die Schöpfung im Anfang weist für Moltmann über die Heilsgeschichte hinaus auf die Vollendung hin. Deshalb ist für ihn nicht die Geschichte der Rahmen der Schöpfung, sondern umgekehrt die Schöpfung (bzw. Natur) der Rahmen der Geschichte. Durch die deutliche eschatologische Akzentuierung gewinnt Moltmann einen universalen Horizont und weitet den Blick auf die ganze Schöpfung - statt nur auf die Menschheit - aus. Seine Position ist damit nicht anthropozentrisch, sondern als "theozentrisch“, da alle Geschöpfe vom Geist her bestehen, im Lobpreis auf ihren Schöpfer ausgerichtet sind (vgl. FN 33) und in ihm ihre Vollendung finden (Moltmann, 1985: 45.149).

Allerdings verwendet Moltmann den Ausdruck „Natur“ nicht äquivok: zum einen erstreckt sich für ihn „Natur" auf die „Schöpfung “ überhaupt, insbesondere als die konkrete, uns erfahrbare Schöpfung, „Natur der Erde“ (Moltmann, 1985: 46). Er verwendet das Wort aber auch - wie im allgemeinen Sprachgebrauch (u. a.) üblich - für die außermenschliche Schöpfung. ${ }^{32}$ So findet er wesentliche Elemente, die ansonsten der menschlichen Geschichte vorbehalten werden, auch in der „Natur“, und zwar „Kontingenz“, „Zukunftsoffenheit“ und „Möglichkeitsfülle“ (Moltmann, 1985: 46). ${ }^{33}$ In seiner Theologie der Evolution zeigt sich, dass er den Menschen und seine Geschichte in das größere Ganze der Schöpfung(-sgemein-

30 Moltmann verweist auf $\$ 41$ der Schöpfungslehre Barths (KD III) (Barth, 1947).

31 Moltmann (1985: 68) denkt die ganze Schöpfung als Einheit, mit dem Ursprung in der Einheit Gottes: „Der Gedanke der Einheit Gottes wird nur in der Vorstellung eines in sich sinnvoll zusammenhängenden Schöpfungsprozesses festgehalten. Dieser Prozess bekommt seinen Sinn von seinem eschatologischen Ziel." Hier will Moltmann sehr wahrscheinlich nicht sagen, die Einheit Gottes hänge von der Einheit des Schöpfungsprozesses $\mathrm{ab}$, sondern er meint, dass aus dem Gedanken der Einheit Gottes eine grundlegende Einheit der Schöpfung folgt.

32 Mit C. Fr. von Weizsäcker spricht Moltmann (1985: 207) sogar - in diesem zweiten Sinne - von einer „Geschichte der Natur“. So liest man: „Auch das Naturgeschehen ist ... ein einmaliger, unumkehrbarer und unwiederholbarer Ablauf mit einer bestimmten Richtung." Moltmann verweist auf Weizsäcker (1952).

33 Dass der Schöpfung ansonsten die Kontingenz abgesprochen wird, ist sicher nicht ganz wahr. Moltmann (1985: 45) legt an anderer Stelle dar, „Natur“ habe - im Unterschied 
schaft) einordnet, das sein eigenes Gewicht und seine eigene Offenheit auf Gott hin hat (Moltmann, 1985: 205ff.). ${ }^{34}$ Die Theologie hat sich nach Moltmann in den vergangenen Jahrhunderten auf das Feld der Geschichte zurückgezogen und die Natur den Naturwissenschaften überlassen.

An einer Stelle in Moltmanns Schöpfungstheologie werden die Lesarten von „Natur“ deutlich, die er verwendet:

Die Theologie muss den Schöpfungsglauben auch aus [der] Überschätzung der [menschlichen] Geschichte befreien. Der Blick auf die ,Geschichte der Natur' ist dabei die eine Perspektive, in der dies geschehen kann. Es fehlt aber auch die andere Perspektive auf die Natur der Erde, in der die menschliche Geschichte geschieht. Und es fehlt endlich die Perspektive auf jene Schöpfung, welche die menschliche Geschichte sowohl zeitlich wie räumlich überragt und überdauert. Gibt es nicht eine natürliche Begrenzung der geschichtlichen Welt der Menschen? (Moltmann, 1985: 46).

Im Folgenden wird deutlich, wie Moltmann die „Natur“ des Menschen selbst - hier also im umfassenden Sinn gebraucht - versteht.

\subsection{Der Mensch als imago mundi bzw. Mikrokosmos}

Der Mensch ist für Moltmann (1985: 197) ein „Mikrokosmos“, der den Makrokosmos repräsentiert. In ihm spiegelt sich für ihn somit die ganze Schöpfung wider. Damit kämen ihm stellvertretende Funktionen zu: „Als ,Bild der Welt' steht der Mensch stellvertretend für alle anderen Geschöpfe vor Gott. Er lebt, spricht und handelt für sie“ (Moltmann, 1985: 197). Im Gedanken vom Menschen als imago mundi kann Moltmann zugleich die Gemeinschaft und Gemeinsamkeit als auch die Unterschiedenheit des Menschen im Blick auf die anderen Geschöpfe darstellen. Der Mensch hat eine besondere Berufung zur Stellvertretung, aber er hat diese gerade (auch) aufgrund seiner besonderen Verbundenheit mit den anderen Geschöpfen! Dieser Gedanke ist wertvoll, obwohl sicher auch Spezifika des Menschen formuliert werden müssen - das möchte Moltmann nicht -, die ihn von den anderen Geschöpfen „abheben“.

zur Geschichte - den „Klang des Zeitlosen, Statischen und Immerwiederkehrenden“ bekommen.

34 Die Schöpfung entwickelt sich nach Moltmann im Verlaufe der Evolution zu immer mehr Gemeinschaft(-sfähigkeit) hin. 
Moltmann (1985: 45) ist es sehr wichtig, dass die Menschen sich zuerst als Teil bzw. „Mitglieder“ der "Schöpfungsgemeinschaft" verstehen. ${ }^{35}$ Der Mensch stehe nicht primär der Natur gegenüber, sondern sei ein „Produkt der Natur“ und solle sich auch "theologisch" - also nicht nur in z. B. naturwissenschaftlicher Betrachtung - als imago mundi verstehen (Moltmann, 1985: 64). Er ist für Moltmann nicht nur ein Produkt der Natur, er „ist“ Natur (Moltmann, 1985: 65). ${ }^{36}$ Der Mensch habe nicht nur einen leiblichen Körper, sondern er sei auch dieser Körper. Moltmann unterstreicht nachdrücklich, es gehe ihm nicht um „romantische Ausflüchte aus der Verantwortung", sondern um die Hervorhebung verdrängter Dimensionen des menschlichen Lebens (Moltmann, 1985: 65). In seinem Verständnis vom Menschen als imago Dei setzt sich dieses Denken fort.

\subsection{Der Mensch als imago Dei}

Für Moltmann ist die Gottebenbildlichkeit nicht primär in den Merkmalen zu suchen und zu finden, in denen sich der Mensch von anderen Geschöpfen unterscheidet, etwa in seiner Seele, durch seinen aufrechten Gang, durch seine Herrschaft auf der Erde oder in der spezifischen Gemeinschaft von Mann und Frau. Das Bild Gottes seien die Menschen in ihrem „ganzen Dasein“, das sich nach Moltmann nicht auftrennen lässt. Bei der Vorstellung der imago Dei gehe es zunächst darum,

35 Moltmann hat mit seinen Aussagen auch den sogenannten Konziliaren Prozess (angestoßen vom ÖRK) beeinflusst. Auf der Versammlung in Dresden formulieren die kirchlichen Delegierten verschiedener Konfessionen gemeinsam:

Die Schöpfungsdarstellung in Gen 1 und die Schöpfungspsalmen (zum Beispiel Ps 104) entwerfen das Bild einer heilen Schöpfungsgemeinschaft in wohlgeordneten Lebensräumen. [...] Der Mensch als Mann und Frau ist einerseits Glied dieser Schöpfungsgemeinschaft, andererseits als Ebenbild Gottes der Haushalter über die ihm anvertrauten Mitgeschöpfe (Ökumenische Versammlung für Gerechtigkeit, Frieden und Bewahrung der Schöpfung. Dresden - Magdeburg - Dresden (1989), Kap. 1.2.4.1). Es fällt - neben dem Ausdruck der „Schöpfungsgemeinschaft“ - auf, dass das „Glied“Sein des Menschen wie bei Moltmann an erster Stelle formuliert wird und so als grundlegend erscheint.

36 Auch der Papst führt in der Enzyklika LS einen ähnlichen Gedanken an: „Man vergisst, dass, der Mensch ... nicht nur sich selbst machende Freiheit ist. Der Mensch macht sich nicht selbst. Er ist Geist und Wille, aber er ist auch Natur.“" (LS 6). Papst Franziskus zitiert hier Papst Benedikt XVI. aus: „Ansprache an den deutschen Bundestag in Berlin (22. September 2011): L'Osservatore Romano (dt.), Jg. 41, Nr. 39 (30. September 2011), S. 5, AAS 103 (2011), S. 664“. 
in welches Verhältnis Gott sich zum Menschen setze (Moltmann, 1985: 226). ${ }^{37}$ Erst sekundär bezeichne sie das Verhältnis des Menschen zu Gott. „Des Menschen Wesen entspringt und besteht in diesem Menschenverhältnis Gottes und nicht in dieser oder jener Eigenschaft, die ihn von anderen Lebewesen unterscheidet“ (Moltmann 1985, S. 226). Gott reflektiere sich im Menschen wie in einem Spiegel, die Menschen würden zu einer „indirekten Offenbarung“ Gottes auf der Erde (Moltmann, 1985: 226f.). ${ }^{38}$ Damit entwickelt Moltmann eine erstaunliche These, wenn er die Gottebenbildlichkeit primär in Gott selbst festmacht. Es muss aber angefragt werden, ob das spezifische Verhältnis, in das sich Gott zum Menschen setzt, nicht doch mit einer Eigentümlichkeit des Menschen in Verbindung steht. Moltmann (1985: 230) leugnet das einerseits, und formuliert dann doch wieder auch Spezifika des Menschen, wenn er z. B. schreibt: „Nur die Menschen kennen den Willen Gottes, nur sie können Gott bewusst loben und preisen“. Sind diese Besonderheiten des Menschen auf ein Menschenverhältnis Gottes zu „reduzieren“?

Moltmann (1985: 227) legt dar, die Menschen stünden als „Stellvertreter“ Gottes „über“ den anderen Geschöpfen und herrschten über sie, zweitens seien sie für Gott ein „Gegenüber“, mit dem er kommunizieren wolle, und schließlich seien sie die „Erscheinung“ seiner Herrlichkeit auf der Erde. ${ }^{39}$ Sind die Menschen als imago mundi für Moltmann Stellvertreter aller Geschöpfe, so sind sie im Gegenzug als imago Dei Stellvertreter Gottes gegenüber den anderen Geschöpfen! Hier könnte zwar eine Überordnung des Menschen über die anderen Geschöpfe herausgelesen werden („über den anderen Geschöpfen“), faktisch aber legt Moltmann in keiner

37 Aus diesem Gedanken heraus ist Moltmann - abweichend von der reformatorischen Tradition - der Auffassung, das Bild Gottes sei durch die Sünde nicht zerstört:

Die menschliche Sünde vermag wohl das Gottesverhältnis des Menschen zu verkehren, nicht aber das Menschenverhältnis Gottes. ... . Darum wird der Sünder subjektiv ganz und gar Sünder und gottlos. Er bleibt darum aber zugleich ganz und gar Gottes Bild und wird diese Bestimmung nicht los, so lange Gott sie festhält und ihm treu bleibt. (Moltmann, 1985: 238)

Moltmann schreibt dann aber auch in Bezug auf das Gottesverhältnis des Menschen, es gehe in der Sünde nicht verloren: „Wie das Böse nur am Guten erscheint, so kann auch die Sünde nur etwas pervertieren, was Gott geschaffen hat, es aber nicht vernichten. Sünde ist die Perversion des Gottesbeziehung des Menschen, nicht ihr Verlust.“ (Moltmann, 1985: 239)

38 Man kann hier anfragen, ob nicht doch - wenn auch möglicherweise sekundär - bestimmte Merkmale des Menschen zur Gottebenbildlichkeit gehören. Gott kann sich ja nur in einem Wesen spiegeln, das er so geschaffen hat, dass dies möglich wird.

39 Hierzu Moltmann (1985: 234): „Als irdisches Bild Gottes spiegeln sie [die Menschen] die Herrlichkeit des Schöpfers wider. Sie sind nicht nur Beauftragte, sondern auch die Erscheinungsweise Gottes in seiner Schöpfung." 
Weise dar, wie diese Überordnung verstanden werden könnte. Er deutet nämlich den Herrschaftsauftrag in Gen 1,28f., in dem er zwei verschiedene Aufträge erkennt, einerseits als Reglementierung, nämlich zur rein vegetarischen Ernährung des Menschen (aber auch der Tiere!), andererseits als einen Dienstauftrag, nämlich als "Aufgabe eines Friedensrichters" in Bezug auf die Tiere (Moltmann, 1985: 230). ${ }^{40}$ Moltmann (2010: 165) möchte Menschen und anderen Lebewesen Rechte zugestehen, versteht die Schöpfungsgemeinschaft somit als „Rechtsgemeinschaft“. Er kann aber zu wenig zeigen, wie hier Abstufungen zwischen dem Menschen und anderen Geschöpfen vorgenommen werden könnten. Alle Lebewesen seien „Bundespartner Gottes ${ }^{\text {“41 }}$ und müssten auch untereinander einen Bund schließen (Moltmann, 2010: 163). Dies kann nur metaphorisch gemeint sein, da ja die anderen Lebewesen faktisch nicht dazu in der Lage sind, einen Bund zu schließen.

Es fällt schwer, diese Gedanken direkt auf ethische Fragestellungen anzuwenden. Was bei Moltmann positiv begegnet, ist eine Wertschätzung aller Geschöpfe, aller Lebewesen, und damit ein Umgang mit ihnen, der anteilnehmend und auf mögliche „Kooperation“ angelegt ist. Darin trifft er sich mit dem Denken des Papstes (LS 11.77.246.) in der neuen Enzyklika, der von einer „Zärtlichkeit“ Gottes gegenüber allen Geschöpfen spricht, welcher der Mensch entsprechen soll, und von der Gemeinschaft der geschwisterlich strukturierten Schöpfung (s. o.).

Zusammenfassend kann gesagt werden, dass Moltmann durch seine gemeinschaftliche und eschatologisch geprägte Denkweise ein neues, umfassendes Verständnis der Natur entwickelt, in welchem der Mensch vor allem als ein Glied unter anderen innerhalb der Schöpfungsgemeinschaft erscheint.

40 Moltmann beruft sich an dieser Stelle auf Steck (1975; 1978: 78ff.).

41 Moltmann nimmt hier Bezug auf den Noachbund und will herausstellen, dass dieser Bund nicht „durch die Menschen geht, sondern ... die Erde selbst in eine direkte Beziehung zu Gott [bringt]“ (Moltmann, 2010: 132, auch S. 163ff.). Moltmann (2010: 128ff.) versteht sogar die Erde als Ganze entsprechend der „Gaja-Theorie“ als eine Art großen „Organismus“. Es bleibt unklar, wie wörtlich er diese Theorie nimmt, da er immer wieder von einem Vergleich spricht und metaphorisch redet. 


\section{Die "Natur" unter der menschlichen Herrschaft und ihre Befreiung}

\subsection{Krise des ganzen Lebenssystems - mangelnde Leidensfähigkeit}

Ein Grundthema Moltmanns ist die Gegenüberstellung von einseitiger Herrschaft und Machtausübung auf der einen und einer gemeinschaftlichen Sicht auf der anderen Seite. ${ }^{42}$ Die „ökologische Krise“ deutet er somit als „Herrschaftskrise“ im Zusammenhang einer größeren, umfassenden „Krise des ganzen Lebenssystems der modernen industriellen Welt“ (Moltmann, 1985: 36). Diese Krise betreffe nicht nur die Umwelt des Menschen, sondern zutiefst diesen selbst. Das Verhältnis des Menschen zur natürlichen Umwelt sei durch die Technologien bereits dauerhaft gestört. ${ }^{43}$ Das menschliche Verhalten bezüglich der Technologien führt Moltmann auf Grundwerte und Grundüberzeugungen zurück, die die Gesellschaften regulierten (Moltmann, 1985: 37). Diese wiederum stammten „,aus fundamentalen Gewissheiten der Menschen über den Sinn und die Bestimmung ihres Lebens" (Moltmann, 1985: 37). Weil die ökologische Krise also ihre Wurzeln in solchen (falschen bzw. destruktiven) Grundüberzeugungen der Menschen habe, sei sie nicht losgelöst zu betrachten, sondern zeigt sich als Teil der „Krise des ganzen Lebenssystems“ (Moltmann, 1985: 36) ${ }^{44}$ Er formuliert prägnant: „Die natürliche Umwelt der Menschen kann nicht isoliert von der sozialen Umwelt begriffen werden. Jene Prozesse,

42 Dies zeigt sich z.B. schon ganz deutlich in seiner Trinitätstheologie, wo er sich immer wieder gegen ein „monarchianisches“ (auch „monotheistisches“), herrschaftliches Verständnis Gottes ausspricht und das Verständnis Gottes als perichoretische Gemeinschaft ohne Über- und Unterordnung dagegenhält (z. B. Moltmann, 1980: 144ff.; 207ff.)

43 Vgl. die kritischen Gedanken von Papst Franziskus zum „technokratischen Paradigma“ (LS 101ff.). „Man neigt zu der Ansicht, ,jede Zunahme an Macht sei einfachhin ,Fortschritt'; Erhöhung von Sicherheit, Nutzen, Wohlfahrt, Lebenskraft, Wertsättigung', als gingen die Wirklichkeit, das Gute und die Wahrheit spontan aus der technologischen und wirtschaftlichen Macht selbst hervor.“ (LS 105, zit. nach Guardini 1965, S. 87)

44 Moltmann (1985: 38) bezieht sich vor allem auf die Situation in den großen Städten und spricht von „Ängsten und Aggressionen“ der Menschen. Er schreibt außerdem: „Überall sind unkontrollierte Wachstumsprozesse entstanden: das Wachstum der Populationen, das industrielle Wachstum, das Wachstum der Umweltbelastung, das Wachstum des Energieverbrauchs, das Wachstum der Reizüberflutung und der seelischen Labilität der Menschen.“ (Moltmann, 1985: 42) 
die zerstörend in die menschliche Umwelt eingreifen, haben ihre Ursachen in den ökonomischen und sozialen Prozessen" (Moltmann, 1985: 37). ${ }^{45}$

Eine Abkehr von der Krise sei also nur möglich, wenn man an diesen Ursachen ansetze! Dazu trägt Moltmann selbst durch seine Theologie bei. Die Normen der Gesellschaften prägten sogar das Unterbewusstsein des Menschen. Wie ein einzelner Organismus, so entwickle auch eine ganze Gesellschaft eine "Selbstimmunisierung" (Moltmann, 1985: 38). Entsprechend gebe es eine „Abwehr der ökologischen Krise“ in der aktuellen Gesellschaft (der 80er Jahre), die dieselbe verharmlose. „Diese Verharmlosungen verdrängen mit dem Schmerz auch die notwendige Umstellung des ganzen Lebenssystems. Der Effekt liegt in der wachsenden Apathie der Menschen gegenüber dem langsamen Sterben der Natur" (Moltmann, 1985: 38). Damit bedrohe der Mensch bzw. die Gesellschaft sich aber auf Dauer selbst, denn nur ein leidensfähiges System sei auch lernfähig und damit überlebensfähig (Moltmann, 1985: 38f., 1985: 211ff.). In seinem Werk zur Eschatologie, zehn Jahre nach „Gott in der Schöpfung“ erschienen, spricht Moltmann dann nicht mehr von der „ökologischen Krise“ - „Krise“ im Sinne einer Zeit der Entscheidung! - , sondern bereits von der „ökologischen Katastrophe“. Es handle sich um eine „langsame, aber sichere und irreversible Katastrophe" (Moltmann, 1995: 234). Natürlich ist er weiterhin der Überzeugung, dass wichtige Entscheidungen für die Menschheit anstehen.

Es wurde schon weiter oben deutlich, dass Moltmann die Schöpfungsgemeinschaft (auch) als Leidensgemeinschaft ${ }^{46}$ versteht, wobei gerade der Christ dieses Leiden der Geschöpfe im „messianischen Licht“, im Geist Christi, vertieft wahrnimmt.

45 Dies ist auch eine Grundthese von Papst Franziskus in der aktuellen Enzyklika Laudato si`! Christian Link hebt diese Einsicht Moltmanns in den Zusammenhang von natürlicher und sozialer Umwelt in seiner Rezension positiv hervor. Er spricht von dem wichtigen „methodischen Grundsatz ..., dass Natur und Geschichte in einem untrennbaren Zusammenhang stehen und sich nicht wie im 19. Jahrhundert gegeneinander definieren lassen“. (Link, 1987: 85). Bereits mit diesen „Weichenstellungen“verlasse Moltmann die „moderne anthropozentrische Auffassung“ (Link, 1987: 85).

46 Das Thema des Mit-Leidens ist Moltmann zentral, auch und gerade für sein Gottesbild. Gott sei nicht apathisch - wie ihn die griechische Philosophie und in der Folge auch die theologische Tradition vornehmlich verstanden habe - sondern er sei, wie sich bereits im AT zeige, ein mit-leidender, anteilnehmder Gott (Moltmann 1980, S. 36ff.). Entsprechend verwirklicht sich auch Menschsein nach Moltmann (1980: 36ff.) u. a. in der Fähigkeit zum Mit-Leiden, die für ihn eng mit der Liebesfähigkeit verbunden ist. Das Leiden der Natur (außerhalb des Menschen) und des menschlichen Leibes beschreibt Moltmann nicht sehr differenziert. Er spricht dabei z. B. - mit Bezug auf den Römerbrief - von der „Knechtschaft“, „Versklavung“ oder „Vergänglichkeit“ der Schöpfung. An keiner Stelle in seinem Werk über die Schöpfung bezieht sich Moltmann ausführlich auf den Sündenfall des Menschen oder auf die Gründe für die Knechtschaft der ganzen Schöpfung. An einer Stelle liest man: „Was in diesem Zustand als ,Natur erkannt wird, ist weder ein reiner 
„Was von den Glaubenden im Heiligen Geist erfahren wird, führt sie in die Solidarität mit der ganzen Kreatur hinein. Sie leiden mit der Natur unter der Macht der Vergänglichkeit und hoffen für die Natur auf die Offenbarung der Freiheit.“ (Moltmann, 1985: 113) $)^{47}$

\section{2 „potentia absoluta“ - Macht über die Natur}

Die modernen Zivilisationsformen sind nach Moltmann (1985: 40) auf Entwicklung, Expansion und Eroberung ausgerichtet. Als Faktor der Entwicklung dieser Art von Zivilisation macht Moltmann das „Gottesbild der Renaissance und des Nominalismus“ aus. Die hervorragende Eigenschaft Gottes sei hier seine „potentia absoluta" gewesen. Dem habe sich der Mensch, der sich als sein Ebenbild verstand, anzugleichen versucht! „Wie kann aber der Mensch Macht gewinnen, um seinem Gott ähnlich zu werden? Durch Wissenschaft und Technik ..."(Moltmann, 1985: 41). Als Paradebeispiel nennt Moltmann Descartes mit seinem vielzitierten Diktum, das Ziel der Naturwissenschaften sei der Mensch als „maître et possesseur de la nature“ (Moltmann, 1985: 41, zit. nach Descartes, 1948: 145). Moltmann (1985: 31) hält den Machtgewinn für das erkenntnisleitende Interesse in den Naturwissenschaften, und bereits deren Forschung - noch vor der Anwendung - sei „machtförmig“. 48 Schon die Methode der Objektivierung der Natur und ihrer Systeme ist für ihn eine Strategie ihrer Unterwerfung. Der Mensch werde dabei als Subjekt der Natur als Objekt gegenübergestellt. Die Objektivierung und Gegenüberstellung wiederum führe zur Ausbeutung. ${ }^{49}$ Eine wichtige Schlussfolgerung Moltmanns lautet:

Urstand, ein paradiesischer Garten Eden, noch das Ende aller Dinge, die Vollkommenheit, sondern ein Schicksal der Schöpfung ... “ (Moltmann, 1985: 53f.). Was bedeutet, was ist dieses „Schicksal“ der Schöpfung? Es bleibt unklar, inwieweit das Leiden und die Vergänglichkeit der Natur bzw. Schöpfung insgesamt mit der menschlichen Sünde oder dem Sündenfall zu tun haben. In seinem Werk zur Trinitätstheologie weist Moltmann ausdrücklich darauf hin, dass die „Erfahrung des Leidens“ deutlich hinausreiche über die „Erfahrung von Schuld“. Sie habe ihre „Wurzeln in den Grenzen der geschaffenen Welt selbst“" (Moltmann, 1980: 66).

47 Moltmann bezieht sich hier wiederum auf Röm 8, $19 \mathrm{ff}$.

48 Diesen Ausdruck zitiert Moltmann (zustimmend) aus: Weizsäcker, 1977: $253 \mathrm{ff}$.

49 Vgl. die Ausführungen von Papst Franziskus zum „technokratischen Paradigma“: Nach diesem Paradigma tritt eine Auffassung des Subjektes hervor, das im Verlauf des logisch-rationalen Prozesses das außen liegende Objekt allmählich umfasst und es so besitzt. Dieses Subjekt entfaltet sich, indem es die wissenschaftliche Methode mit ihren Versuchen aufstellt, die schon explizit eine Technik des Besitzens, des Beherrschens und des Umgestaltens ist. (LS 106) 
„Er [der Mensch] ist nicht mehr Glied der Schöpfungsgemeinschaft, sondern stellt sich ihr [der Natur] als Herr und Eigentümer gegenüber. Er kann sich folglich nicht mehr leiblich und natürlich identifizieren, sondern wird zum alleinigen Subjekt von Erkenntnis und Wille“ (Moltmann, 1985: 41).

Descartes sah den Leib nach Moltmann als eine Maschine an, die auch unabhängig vom Geist genau so existieren könnte (Moltmann, 1985: 254). ${ }^{50}$ Das Verhältnis von Geistsubjekt (res cogitans) und zugehörigem Körper denke er als „einseitiges Herrschafts- und Eigentumsverhältnis“"(Moltmann, 1985: 255), also exakt entsprechend dem Verhältnis des Subjekts zur ausgedehnten Welt, der res extensa, überhaupt. Der Körper werde, in dieser Weise im Gegensatz zur Seele definiert, „in eine vermeintliche Geistlosigkeit" verbannt (Moltmann, 1985: 255). ${ }^{51}$

Entsprechend seinem spezifisch akzentuierten Gottesbild (als nicht hierarchische Gemeinschaft, die sich ebenso zur Schöpfung verhält) versteht Moltmann auch das Verhältnis des Menschen zur Schöpfung und zu seinem eigenen Leib in deutlichem Kontrast zu diesem neuzeitlichen Denken. Statt des gegenwärtigen „Herrschaftwissens" sucht er nach einem „Gemeinschaftswissen“, einem Erkennen, das Gemeinschaft und „Frieden“ (Moltmann, 1985: 35) zwischen Mensch und Natur stiftet (Moltmann, 1985: 47).

Verstehen wir Gott nicht mehr auf monotheistische Weise als das eine, absolute Subjekt, sondern auf trinitarische Weise als die Einheit des Vaters und des Sohnes und des Geistes, dann können wir sein Verhältnis zu der von ihm geschaffenen Welt auch nicht mehr als ein einseitiges Herrschaftsverhältnis auffassen, sondern müssen es als ein vielschichtiges und mehrstelliges Gemeinschaftsverhältnis verstehen. Dies ist der Grundgedanke der nicht-hierarchischen, dezentralisierten, genossenschaftlichen Theologie. (Moltmann, 1985: 16).

Auch in seinem Werk „Ethik der Hoffnung“ von 2010 formuliert Moltmann (2010: 153) Ähnliches:

Technologien und Naturwissenschaften werden immer aus bestimmten menschlichen Interessen heraus entwickelt. Es gibt sie nicht wertfrei. Interessen gehen ihnen voraus, leiten sie und nehmen ihre Ergebnisse in Dienst. Diese menschlichen Interessen werden ihrerseits von Grundwerten und Überzeugungen einer Gesellschaft reguliert. ... Welche Interessen und Werte regieren die moderne Zivilisation? Es ist offenbar der Wille zur Herrschaft, der die modernen Menschen zur Machtergreifung über die Natur der Erde und ihre eigene leibliche Natur ergreift.

50 Moltmann, 1985: 254. Moltmann zitiert Descartes, 1948: 33 (6. Meditation).

51 Moltmann (1985: 256) zeigt in Bezug auf Karl Barth, dass bei diesem das Verhältnis von Seele und Leib dem Herrschaftsverhältnis Gottes zur Welt entspreche: „In ihrer Herrschaft über den Leib nimmt die Seele unmittelbar an der Herrschaft Gottes über seine Welt teil.“ 
Analog denkt sich Moltmann das Verhältnis von Leib und Seele als ein partnerschaftliches, gemeinschaftliches, nicht hierarchisches. Er spricht sogar von einem „Bund“ und einer „Übereinkunft" zwischen beiden Aspekten des Menschen (Moltmann, 1985: 263). Für ihn ist somit die Wiederentdeckung der Ganzheit der menschlichen Person in Leib und Seele ein entscheidender Beitrag zur Überwindung der ökologischen Krise, die er auch auf das Verhältnis des Menschen zum eigenen Leib bezieht. $^{52}$

\section{$6 \quad$ Kraft aus der gemeinsamen Zukunft!}

Im Gesamtblick auf die dargestellten Thesen Moltmanns - die nur ein Ausschnitt aus seiner reichen und differenzierten Schöpfungstheologie sein können -, zeigt sich einerseits seine starke Orientierung am Gedanken der Gemeinschaft (in Gott selbst, zwischen Gott und Schöpfung, innerhalb der Schöpfung), andererseits sein hermeneutischer Zugang über die Eschatologie, die verheißene Vollendung der Schöpfung. Die Kraft für den Umgang mit der Theodizeefrage, für die aktuelle ethische Orientierung, kommt bei ihm dabei aus dieser umfassenden eschatologischen Hoffnung, die sich auf die Vollendung der Schöpfungsgemeinschaft mit allen ihren Gliedern richtet. Der Mensch wird als Glied in diese Schöpfungsgemeinschaft eingeordnet.

Wie bereits zu Beginn gesehen, ist der Gedanke der Gemeinschaftlichkeit in der Schöpfung auch für Papst Franziskus wichtig. Papst Franziskus begründet diesen Gedanken ebenfalls durch die Gemeinschaft („Communio") in Gott selbst, akzentuiert ihn jedoch anders als Moltmann, indem er den Vater in den Mittelpunkt stellt, durch dessen Ursprünglichkeit und in Hinordnung auf den alle Geschöpfe zu „Geschwistern" verbunden seien. Aber auch er lenkt den Blick auf die Vollendung der Schöpfung:

52 Damit hat Moltmann sicher Wichtiges beobachtet und gesagt. Man muss ihm dennoch entgegenhalten, dass er das nicht-hierarchische, "genossenschaftliche“ (s.o.) Denken übertreibt. Es gibt durchaus eine „gesunde“ und angemessene Form von Herrschaft und Überordnung des Menschen über die anderen Geschöpfe, der Seele über den Leib, wie auch Gott der Schöpfung per se, als Schöpfer, übergeordnet ist, selbst wenn er zu ihr freiwillig in ein Gemeinschaftsverhältnis eintritt. Analog kann sich der Mensch der ihn umgebenden Natur als übergeordnet verstehen, ohne dabei ein gemeinschaftliches Verständnis auszuschließen! 


\begin{abstract}
Das Neue Testament spricht zu uns nicht nur vom irdischen Jesus und seiner so konkreten und liebevollen Beziehung zur Welt. Es zeigt ihn auch als den Auferstandenen und Verherrlichten, der mit seiner allumfassenden Herrschaft in der gesamten Schöpfung gegenwärtig ist: ,Gott wollte mit seiner ganzen Fülle in ihm wohnen, um durch ihn alles zu versöhnen. Alles im Himmel und auf Erden wollte er zu Christus führen, der Friede gestiftet hat am Kreuz durch sein Blut.' (Kol 1-19-20) Das versetzt uns ans Ende der Zeiten, wenn der Sohn dem Vater alles übergibt und Gott alles in allem ist (vgl. 1 Kor 15,28). Auf diese Weise erscheinen uns die Geschöpfe dieser Welt nicht mehr als eine bloß natürliche Wirklichkeit, denn geheimnisvoll umschließt sie der Auferstandene und richtet sie auf eine Bestimmung der Fülle aus. Die gleichen Blumen des Feldes und die Vögel ${ }^{53}$, die er mit seinen menschlichen Augen voll Bewunderung betrachtete, sind jetzt erfüllt von seiner strahlenden Gegenwart." (LS 100). ${ }^{54}$
\end{abstract}

Diese Sätze des Papstes überraschen, denn sie scheinen - wie Moltmann - von der Erlösung der individuellen - nicht-menschlichen - Geschöpfe auszugehen, ${ }^{55}$ was bisher nicht offizielle Lehre der römisch-katholischen Kirche ist. ${ }^{56}$

53 Möglicherweise (!) müsste man hier sogar mit „Dieselben ...“ übersetzen; das wird inhaltlich nicht ganz deutlich. Im Italienischen heißt es: „Gli stessi..." (was beide Bedeutungen haben kann!) und im Englischen: „The very..... Dann müsste man das ,jetzt“ auf die eschatologische Wirklichkeit beziehen, was auch inhaltlich passen könnte, nachdem Papst Franziskus zuvor schreibt: „Das versetzt uns ans Ende der Zeiten...“.

54 Mit diesem Gedanken schließt Franziskus seine Ausführungen zum „Evangelium von der Schöpfung“, welche das zweite Kapitel seiner Enzyklika bilden. Durch diese Schlussstellung, die natürlich auch inhaltlich bedingt ist, kommt den Sätzen eine besondere Bedeutung zu. Vgl. auch das „Christliche Gebet mit der Schöpfung“, mit welchem die ganze Enzyklika schließt und dem somit auch zentrale Bedeutung zukommt. Hier liest man über Jesus, den „Sohn Gottes“: „Jetzt lebst du in jedem Geschöpf mit deiner Herrlichkeit als Auferstandener." (LS Nr. 246) Es wird der Auferstehung also eine Bedeutung für jedes Geschöpf zugesprochen. Implizit ist ausgesagt, dass jedes Geschöpf Anteil an der Auferstehung Jesu erhält.

55 Kardinal Reinhard Marx sagte in einer Veranstaltung der Katholischen Akademie in Bayern, welche sich der neuen Enzyklika widmete:

Der Papst löst sich von einem gewissen Anthropozentrismus, den wir immer noch haben, auch in unserer abendländischen Theologie; etwa in der Formulierung, die Geschöpfe sind nicht alle für den Menschen da; sie sind auch um ihrer selbst willen da. ... . Das ist auch eine Einladung, theologisch neu darüber nachzudenken, was Schöpfung bedeutet. Teilhard de Jardin hat vielleicht als Einziger versucht, die Schöpfungslehre auch im Sinne dessen zu entfalten, was es eigentlich für die Erlösung heißt. Er weist auf den auferstandenen Christus hin. („Laudato si““2015: 8).

56 Der „Katechismus der Katholischen Kirche“ von 1993 (Libreria Editrice Vaticana 1993) spricht vom „neuen Himmel und der neuen Erde“, von der Erneuerung des ganzen „Weltalls“ bzw. der „materiellen Welt" und des „,sichtbaren Universums“ (KKK 1042ff.). Es wird nicht ausdrücklich ausgeschlossen, aber auch nicht positiv benannt, ob individuelle Geschöpfe erlöst werden. (Von ihnen ist gar nicht die Rede.) In einem 
Die Liebe Gottes zu allen Geschöpfen, die sich in seinem Erlösungswillen zu den Geringsten ${ }^{57}$ derselben manifestiert, kann ein ungeheures ethisches Potential bieten, denn sie lässt die ökologischen Fragen - selbst die, die sich kaum auf den Menschen beziehen - als originär geistliche und damit auch im engeren Sinne kirchliche Fragen erscheinen. Die Geschöpfe sind nicht nur „natürlich“, wie der Papst ausführt, sondern selbst auf eine übernatürliche Wirklichkeit, auf ihre individuelle Vollendung, ausgerichtet.

Weiterhin schreibt Papst Franziskus:

Das Ziel des Universums liegt in der Fülle Gottes, die durch den auferstandenen Christus - den Angelpunkt des universalen Reifungsprozesses - schon erreicht worden ist. ${ }^{58}$ So fügen wir ein weiteres Argument hinzu, um jede despotische und verantwortungslose Herrschaft des Menschen über die anderen Geschöpfe abzulehnen. Der letzte Zweck der anderen Geschöpfe sind nicht wir. Doch alle [!] gehen mit uns und durch uns voran auf das gemeinsame Ziel, das Gott ist, in einer transzendenten Fülle, wo der auferstandene Christus alles umgreift und erleuchtet. Denn der Mensch,

Zitat aus Lumen Gentium (LG 48) wird die innigste Verbundenheit des Menschen mit der gesamten Welt zum Ausdruck gebracht, welche durch ihn auf das Ziel der Erneuerung in Christus zugehe (KKK 1042). Da die Welt nun einmal aus Individuen besteht, könnte man schließen, dass die individuellen Geschöpfe in ihrer Verbundenheit mit dem Menschen erlöst werden. Das wird jedoch im Katechismus nicht ausdrücklich vertreten. - Der deutsche Erwachsenenkatechismus kennt nur die Unterscheidung des Menschen von der „materiellen Welt“, welche auch endgültig verklärt werden solle (Deutsche Bischofskonferenz, 1985: 106f.).

57 So liest man in „Laudato si ““:

Die Liebe Gottes ist der fundamentalste Beweggrund der gesamten Schöpfung: ,Du liebst alles, was ist, und verabscheust nichts von dem, was du gemacht hast; denn hättest du etwas gehasst, so hättest du es nicht geschaffen.' (Weish 11,24). Jedes Geschöpf ist also Gegenstand der Zärtlichkeit des Vaters, der ihm einen Platz in der Welt zuweist. Sogar das vergängliche Leben des unbedeutendsten Wesens ist Objekt seiner Liebe, und in diesen wenigen Sekunden seiner Existenz umgibt er es mit Wohlwollen. (LS 77) Der Papst ist hier vermutlich inspiriert von Gedanken des heiligen Bonaventura, der über Franziskus schreibt: „Eingedenk dessen, dass alle Geschöpfe ihren letzten Ursprung in Gott haben, war er von noch überschwänglicherer Zuneigung zu ihnen erfüllt. Auch die kleinsten Geschöpfe nannte er deshalb Bruder und Schwester." Der Papst zitiert dies (mit der Angabe: Legenda Maior, VIII, 6: FF 1145, dt. Ausg.: ebd., S. 736) in LS 11, als Teil der Einleitung der Enzyklika.

58 Papst Franziskus weist hier in einer Fußnote auf den ,entsprechende[n] Beitrag von Pierre Teilhard de Chardin SJ" hin! Teilhard de Chardin erfährt hier eine Würdigung, die er zu Lebzeiten vermisst hat, und wird aus einer Randposition heraus zu einem bedeutenden Zeugen des Glaubens erhoben. 
der mit Intelligenz und Liebe begabt ist und durch die Fülle Christi angezogen wird, ist berufen, alle Geschöpfe [! $]^{59}$ zu ihrem Schöpfer zurückzuführen. (LS 83) ${ }^{60}$

Man könnte sagen: das tiefste Argument, das Papst Franziskus für die Achtung der ganzen Schöpfung anführt, ist ein originär theologisches, nämlich die Liebe des göttlichen Vaters zu allen Geschöpfen und sein Erlösungswille für sie. Damit trifft sich der Papst in einem zentralen Punkt mit den Gedanken Moltmanns, der seine Ethik vor allem aus dem Gedanken der Gott (im Blick auf die Vollendung) entsprechenden, von ihm gewollten und geliebten Gemeinschaft der Schöpfung begründet, in welcher kein Geschöpf verloren geht.

Jürgen Moltmann kann in der Reflexion auf die „Schöpfungsgemeinschaft“ nur schwer eine differenzierte Form von Gemeinschaft denken, in der die Menschen den anderen Geschöpfen (und ihrem Leib) zwar übergeordnet sind, und dennoch in einem "gemeinschaftlichen" Verhältnis zu ihnen (in einem weiten Sinn) stehen können. ${ }^{61}$ So kann sein Denken eine gute Ergänzung erfahren durch das, was Papst Franziskus deutlich formuliert, nämlich dass die Einheit der Schöpfung in ihrem gemeinsamen Grund, dem Vater, besteht (welcher der Schöpfung übergeordnet ist).

59 Beide Hervorhebungen durch Ausrufezeichen in Klammer stammen von der Verfasserin.

60 Der Papst formuliert hier gleichsam eine „priesterliche“ Funktion der Menschen in Bezug die anderen Geschöpfe, wie sie in der orthodoxen Tradition gedacht wird und auch im Denken Moltmanns aufscheint. Moltmann (1985: 84) sagt über den stellvertretenden Lobpreis der Menschen oder den oben dargestellten Gedanken, dass die Menschen zu „Friedensrichtern“ bestimmt sind (Moltmann, 1985: 230). An einer Stelle schreibt er: „Darum wartet die geknechtete Kreatur nicht unmittelbar auf die Erscheinung Christi in Herrlichkeit, sondern auf die Offenbarung der Freiheit der Kinder Gottes in Christi Erscheinung. Durch menschliche Freiheit soll sie erlöst werden." (Moltmann, 1985: 82).

61 Es wurde bereits angedeutet (vgl. FN 15), dass dies mit den Akzentsetzungen in seiner Trinitätslehre zusammenhängt. Auch wenn Moltmann an einzelnen Stellen darlegt, wie sich Vater, Sohn und Geist zur Schöpfung verhalten, dominiert bei ihm - zumindest im Werk „Gott in der Schöpfung“ - doch der pneumatologische Zugang. Obwohl er Vater, Sohn und Geist als perichoretische Gemeinschaft in engem Lebensaustausch versteht, treten die Personen im Blick auf die Schöpfungstheologie zu weit ,auseinander“. Moltmann spricht vom kosmischen Geist und von kosmischer Christologie (Moltmann, 2010: 158f.), kann beide aber zu wenig verknüpfen. Durch die zu geringe Berücksichtung des Aspektes der Ursprungsbeziehungen (Hervorgänge von Sohn und Geist aus dem Vater) in der Konzentration auf die Gemeinschaftlichkeit in Gott verschwimmen Moltmann ungewollt die Spezifika der Personen. Die Herausforderung wäre, sowohl in der Trinitätslehre, als auch in der Schöpfungstheologie, Herkunft und Ursprung (die gewissermaßen einseitig sind), auch Überordnung, zusammen zu denken mit einer gemeinschaftlichen Art von Beziehung, die Austausch des Lebens und Wechselseitigkeit bedeutet. Nur so kann letztlich die Einheit Gottes (in seinem einen Wesen), aber auch die Einheit der Schöpfung (in ihrer Herkunft von Gott) gedacht werden. 
Entsprechend lässt sich auch der Mensch als der übrigen Schöpfung (und gewissermaßen seinem eigenen Leib) übergeordnet und dennoch gleichzeitig in einem gemeinschaftlichen Verhältnis zu ihr verstehen. Gerade der Gedanke der Geschwisterlichkeit in der Schöpfung, analog zur Geschwisterlichkeit in der christlichen Gemeinde und unter den Menschen, welchen Moltmann so nicht entfaltet ${ }^{62}$, kann zusammen mit dem Hinweis auf die Vollendung eine große (auch und besonders ethische) Orientierungskraft entfalten. In der Herkunft und Rückbindung an den Vater (durch den Sohn im Heiligen Geist) wird eine tiefe Verbindung der Menschen mit allen Geschöpfen und damit deren „Würde“ und Heiligkeit - mindestens deren eigener Wert - deutlich. ${ }^{63}$

Moltmann hingegen kann den Papst „ergänzen“ bzw. in gewissem Sinne weiter „ermutigen“, was seine starke und ausführliche Orientierung an der gemeinsamen Zukunft der ganzen Schöpfung angeht. Ebenfalls kann dieser von Moltmann „lernen“, mit Blick auf das Kreuz Christi den umfassenden Erlösungswillen Gottes und die Leiden auch der außermenschlichen Geschöpfe wahrzunehmen und ernst zu nehmen, möglicherweise sogar die "Sehnsucht" der ganzen Schöpfung nach Befreiung und Vollendung. ${ }^{64}$

Es bleibt festzuhalten, dass sich zwischen den Gedanken des Papstes und Moltmanns erstaunliche und bemerkenswerte Parallelen zeigen. Beide haben spezifische Akzente, die sich wechselseitig ergänzen können. Durch die Enzyklika erhält Moltmann als reformierter Theologe so eine indirekte Würdigung durch den katholischen Papst, was sicher auch ökumenisch von Bedeutsamkeit ist.

62 Moltmann (1985: 247) legt zwar an einer Stelle sogar dar, dass die Menschen in besonderer Weise der Person des Sohnes entsprechen und nach seinem Bild erlöst vollendet werden. Da seine ganze Schöpfungslehre aber anders akzentuiert ist, weitet er diesen Gedanken nicht auf alle Geschöpfe aus.

63 „Die beste Art, den Menschen auf seinen Platz zu verweisen und seinem Anspruch, ein absoluter Herrscher über die Erde zu sein, ein Ende zu setzen, besteht darin, ihm wieder die Figur eines Vaters vor Augen zu stellen, der Schöpfer und einziger Eigentümer der Welt ist." (LS 75).

64 Der Papst bringt zwar alle Geschöpfe mit der Auferstehung Jesu in Verbindung, schweigt aber über die Bedeutung des Kreuzes in dieser Hinsicht. - Jürgen Moltmann kommt weiterhin das Verdienst zu, das Schöpfungswirken des Geistes Gottes neu herausgestellt zu haben. 


\section{Literatur}

Aung, S. H. (1998). The Doctrine of Creation in the Theology of Barth, Moltmann and Pannenberg: Creation in Theological, Ecological and Philosophical-Scientific Perspective. Regensburg: Roderer.

Baukham, R. (2001). Time and Eternity. In: R. Baukham (Hrsg.), God will be all in all. The Eschatology of Jürgen Moltmann (pp.155-226). Minneapolis: Fortress Press.

Barth, K. (1947). Kirchliche Dogmatik, Bd. III/1: Die Lehre von der Schöpfung. Zollikon-Zürich: EVZ.

Bouma-Prediger, S. (1995). The Greening of Theology: The Ecological Models of Rosemary Radford Ruether, Joseph Sittler, and Jürgen Moltmann. Atlanta: Scholars Press.

Descartes, R. (1692, 1948). Discours de la Méthode. Mainz: Internationaler Universum-Verlag.

Deutsche Bischofskonferenz (Hrsg.) (1985). Katholischer Erwachsenenkatechismus. Erster Band: Das Glaubensbekenntnis der Kirche. Kevelaer: Butzon \& Bercker (u. a.).

Guardini, R. (1965). Das Ende der Neuzeit. 9. Aufl. Würzburg: Werkbund.

Greshake, G. (2001). Der dreieine Gott. Eine trinitarische Theologie. 4. Aufl. Freiburg: Herder.

Haudel, M. (2006). Die Selbsterschließung des dreieinigen Gottes. Grundlage eines ökumenischen Offenbarungs-, Gottes- und Kirchenverständnisses. Göttingen: Vandenhoeck \& Ruprecht.

Laudato sí (2015). Ein Gespräch zur neuen Papst-Enzyklika (Podiumsdiskussion von Kardinal Reinhard Marx, Prof. Dr. Klaus Töpfer und Akademiedirektor Florian Schuller am 2. Juli 2015 in der Katholischen Akademie in Bayern). zur debatte. Themen der Katholischen Akademie in Bayern 7, 8.

Libreria Editrice Vaticana (Hrsg.) (1993). Katechismus der Katholischen Kirche. München: R. Oldenbourg Verlag.

Link, C. (1987). Schöpfung im messianischen Licht. Evangelische Theologie, 47: 83-92.

Manzke, K.-H. (2015). Stellungnahme zur Enzyklika Laudato si von Papst Franziskus (Catholica-Beauftragter der VELKD), 19. Juni 2015. Vereinigte Evangelisch-Lutherische Kirche Deutschlands. http://www.velkd.de. Zugegriffen: 30.10.2015.

Marx, R. (2015). Würdigung der neuen Enzyklika „Laudato si - Über die Sorge für das gemeinsame Haus" durch den Vorsitzenden der Deutschen Bischofskonferenz, Reinhard Kardinal Marx, bei der Pressekonferenz am 18. Juni 2015 in München. Deutsche Bischofskonferenz. http://www.dbk.de. Zugegriffen: 30.10.15.

Moltmann, J. (1980). Trinität und Reich Gottes. Zur Gotteslehre. Gütersloh: Chr. Kaiser.

Moltmann, J. (1985). Gott in der Schöpfung. Ökologische Schöpfungslehre. München: Chr. Kaiser.

Moltmann, J. (1995). Das Kommen Gottes. Christliche Eschatologie. Gütersloh: Chr. Kaiser.

Moltmann, J. (1999). Erfahrungen theologischen Denkens. Wege und Formen christlicher Theologie. Gütersloh: Chr. Kaiser.

Moltmann, J. (2010). Ethik der Hoffnung. Gütersloh: Gütersloher Verlagshaus.

Ökumenische Versammlung für Gerechtigkeit, Frieden und Bewahrung der Schöpfung. Dresden -Magdeburg - Dresden (1989). Stiftung Oekumene - Ökumenischer Informationsdienst Ecunet. http: oikumene.net/regional/dresden/dmd4/index.html. Zugegriffen: 14. August 2015.

Pehar, M. (2006). Schöpfung zwischen Trinität und Eschaton. Die Schöpfungstheologie Jürgen Moltmanns im Gesamtkontext seiner Theologie. Berlin: LIT Verlag. 
Picht, G. (1989). Der Begriff der Natur und seine Geschichte. Stuttgart: Klett-Cotta.

Sekretariat der Deutschen Bischofskonferenz (Hrsg.) (2015). Enzyklika LAUDATO SI' von Papst Franziskus über die Sorge für das gemeinsame Haus (Verlautbarungen des Apostolischen Stuhls, Nr. 202). Bonn: Libreria Editrice Vaticana.

Sicouli, P. C. (2006). Die Kategorie „Neuschöpfung“ und die Modelle der Verhältnisbestimmung von Schöpfung und Neuschöpfung im Werk Jürgen Moltmanns. Catholica, 3: $184-217$.

Steck, O. H. (1975). Der Schöpfungsbericht der Priesterschrift. Göttingen: Vandenhoeck \& Ruprecht.

Steck, O. H. (1978). Welt und Umwelt. Stuttgart: Kohlhammer.

Weizsäcker, C. F. v. (1952). Die Geschichte der Natur. Göttingen: Vandenhoeck \& Ruprecht.

Weizsäcker, C. F. v. (1977). Der Garten des Menschlichen. Beiträge zur geschichtlichen Anthropologie. München: Hanser.

Open Access Dieses Kapitel wird unter der Creative Commons Namensnennung - Nicht kommerziell 2.5 International Lizenz (http://creativecommons.org/licenses/by-nc/2.5/deed. de) veröffentlicht, welche für nicht kommerzielle Zwecke die Nutzung, Verbreitung und Wiedergabe in jeglichem Medium und Format erlaubt, sofern Sie den/die ursprünglichen Autor(en), den Titel des Werks und die Quelle ordnungsgemäß nennen, einen Link zur Creative Commons Lizenz beifügen und im Falle einer Abwandlung durch einen entsprechenden Hinweis deutlich erkennbar machen, dass Änderungen vorgenommen wurden.

Die in diesem Kapitel enthaltenen Bilder und sonstiges Drittmaterial unterliegen ebenfalls der genannten Creative Commons Lizenz, sofern sich aus der Abbildungslegende nichts anderes ergibt. Sofern das betreffende Material nicht unter der genannten Creative Commons Lizenz steht und die betreffende Handlung nicht nach gesetzlichen Vorschriften erlaubt ist, ist auch für die oben aufgeführten nicht-kommerziellen Weiterverwendungen des Materials die Einwilligung des jeweiligen Rechteinhabers einzuholen. 